\title{
CONHECIMENTO, APRENDIZAGEM E INOVAÇÃO EM ORGANIZAÇÕES: UMA PROPOSTA DE ARTICULAÇÃO CONCEITUAL
}

\author{
Antonio Isidro Filho \\ Mestrado em Administração pela Universidade de Brasília - UNB \\ Professor da Universidade de Brasília - UNB \\ antonio.isidro@uol.com.br \\ Tomás de Aquino Guimarães \\ Doutorado em Sociologia pela Universidade de São Paulo - USP \\ Professor Associado da Universidade de Brasília - UNB \\ tomas.aquino.guimaraes@gmail.com
}

\section{RESUMO}

Conhecimento, aprendizagem e inovação em organizações são constructos polissêmicos e sua literatura é fragmentada e dispersa. Ao mesmo tempo, organizações de serviços possuem peculiaridades próprias que requerem um tratamento conceitual diferenciado do que é válido para organizações industriais em geral. Nessa linha, é importante articular teoricamente os conceitos de conhecimento, aprendizagem e inovação em organizações de serviços, objetivo principal desse ensaio. Para alcançar esse objetivo, inicialmente cada constructo é definido, considerando suas especificidades. Em seguida, as interfaces e limites entre os construtos são discutidos, com destaque para similaridades e diferenças teóricas e práticas. À luz da abordagem da Economia do Aprendizado, do Paradigma Tecno-Econômico e da Inovação em Serviços, a articulação entre os três conceitos é apoiada na perspectiva de interação entre diferentes stakeholders. No final são formuladas sugestões para futuras pesquisas que considerem conhecimento, aprendizagem e inovação como conceitos interdependentes pertencentes ao mesmo campo temático.

Palavras-Chave: Conhecimento em Organizações, Aprendizagem em Organizações, Inovação, Inovação em Serviços, Estudos Organizacionais. 


\section{INTRODUÇÃO}

Conhecimento, aprendizagem e inovação são termos comumente empregados para descrever fenômenos individuais e coletivos presentes no ambiente de organizações em geral. Nas últimas décadas, o crescente número de pesquisas acerca dos três constructos pode estar relacionado a diversas variáveis contextuais, como mudanças em políticas de pessoal em virtude de mudanças demográficas; alteração nas relações industriais em conseqüência de mudanças nos valores sociais; adaptação exigida por um ambiente global mais competitivo; novas competências e repertórios comportamentais em função do rápido desenvolvimento tecnológico; eventos políticos que impõem restrições e oportunidades para as organizações; entre outras.

De acordo com Hargadon (2002), estudos que integrem conhecimento, aprendizagem e inovação em organizações constituem uma alternativa segura para a compreensão mais apurada de fenômenos individuais e coletivos que determinam a continuidade, adaptação e mudança em organizações. Apesar de haver consenso de que conhecimento e aprendizagem são condições indispensáveis para a inovação, a maneira como esses conceitos estão relacionados é uma lacuna na literatura de estudos organizacionais.

A compreensão de como conhecimento, aprendizagem e inovação estão associados parece repousar sobre a noção da "lógica inovativa", apresentada por Callon (2007), em que a competitividade sustentável de organizações decorre de relações estabelecidas entre estas, seus stakeholders e outros atores sociais no intuito de gerar ou adotar tecnologias, competências e conhecimentos estruturados que apóiam e resultam em inovações reconhecidas no mercado. Essa noção refere-se às redes tecno-econômicas que representam a dimensão coletiva ou social de processos de inovação e descrevem a organização de mercados, de modo a retratar sua complexidade e dinamismo.

Nos estudos organizacionais pode-se observar um discurso perene sobre a relação entre os constructos mencionados, ditando de que forma as organizações podem inovar por meio de aprendizagem ou de conhecimentos, como a aprendizagem e conhecimento criam condições para inovação, e como uma inovação gera novas aprendizagens e conhecimentos. Os conceitos de conhecimento, aprendizagem e inovação em organizações são comumente caracterizados como polissêmicos e de difícil integração. Em conseqüência, a literatura de cada um e, principalmente, a literatura entre os campos que delimitam esses conceitos é fragmentada e dispersa em diversas áreas das ciências sociais.

Revista de Administração e Inovação, São Paulo, v. 7, n. 2, p. 127-149, abr./jun. 2010 
Dessa forma, o presente ensaio tem por objetivo propor uma articulação teórica entre conhecimento, aprendizagem e inovação em organizações. O texto está estruturado da seguinte maneira: a primeira parte aborda a interface entre conhecimento e aprendizagem em organizações. Em seguida, discute-se a noção de inovação, e, por fim, apresenta-se uma proposta de integração teórica que aproxime esses conceitos e indique caminhos de pesquisa com vistas ao desenvolvimento de uma literatura cumulativa e integrada.

\section{REVISÃO BIBLIOGRÁFICA}

\subsection{CONHECIMENTO E APRENDIZAGEM EM ORGANIZAÇÕES}

Os estudos sobre conhecimento e aprendizagem em organizações começam a se desenhar a partir dos trabalhos de Polanyi (1958), Simon (1979) e March e Simon (1981). Segundo Polanyi (1958), a dimensão mais intrínseca do conhecimento, chamada conhecimento tácito, enfatiza que o conhecimento individual pode ser desestruturado, não codificado e não publicado, bem como diferente de pessoa para pessoa. No entanto, essa dimensão do conhecimento pode ser partilhada com outros indivíduos a partir da articulação da linguagem em um contexto comum de interação. Simon (1979) afirma que o conhecimento tem o papel de determinar antecipadamente as consequências das escolhas ou alternativas desenvolvidas por indivíduos nas organizações frente ao problema da adaptação exigida pelo ambiente, conforme destacam March e Simon (1981). Nesse sentido, o comportamento adaptativo da organização necessita de normas e processos estáveis que garantam a adaptação em curto prazo (solução de problemas) ou em longo prazo (aprendizado). Aqui ocorre o elo entre conhecimento e aprendizagem visto que, segundo Argyris e Schön (1996), o conhecimento está na mente dos indivíduos e também pode ser encontrado em rotinas e práticas identificadas e descritas por membros organizacionais, resultando em solução de problemas e aprendizado em organizações.

$\mathrm{Na}$ literatura de estudos organizacionais, especialmente em campos como Psicologia e Sociologia, conhecimento e aprendizagem são considerados áreas de estudos distintas, mas com similaridades epistemológicas. Segundo Chiva e Alegre (2005), existem duas principais abordagens teóricas sobre conhecimento e aprendizagem, uma com ênfase cognitiva e outra com ênfase social. Esses autores, bem como Von Krogh (1998), Spender e Grant (1996), e Grant (1996), mostram que o conhecimento na visão cognitiva refere-se a um ativo organizacional que pode ser mensurado, codificado, armazenado e transmitido para outros indivíduos. A

Revista de Administração e Inovação, São Paulo, v. 7, n. 2, p. 127-149, abr./jun. 2010 
aprendizagem na visão cognitiva, segundo Chiva e Alegre (2005), refere-se ao processo que permite a organização processar, interpretar e responder a informações advindas de seu ambiente interno e externo. Inclui a aplicação de princípios de aprendizagem individual na tentativa de identificar processos-chave e padrões de aprendizagem comuns aos diversos contextos organizacionais.

A visão social de conhecimento refere-se às condições organizacionais que podem influenciar e incentivar sua criação e compartilhamento. Envolve processos individuais e sociais de criatividade, inovação, motivação e comunicação. A realidade é socialmente construída e baseada nas interações entre indivíduos (Chiva; Alegre, 2005; Von Krogh, 1998). A aprendizagem na visão social, conforme Lave e Wenger (1991), Gherardi e Nicolini (2003), Chiva e Alegre (2005), Elkjaer (2005) refere-se à forma como indivíduos interpretam ou atribuem significado às suas experiências no trabalho e considera os indivíduos como seres sociais que aprendem e constroem coletivamente a compreensão do contexto que os cerca. Nessa linha, as organizações são consideradas sistemas sociais de interação entre indivíduos.

Com base no exposto até aqui, pode-se observar que conhecimento e aprendizagem em organizações estão imbricados enquanto constructos que explicam mudanças organizacionais a partir de solução de problemas e aquisição de capacidades de resposta às demandas contextuais. Entretanto, a partir da década de 1990 observa-se a proliferação de estudos, conceitos e paradigmas endereçados à descrição de processos de aprendizagem, bem como da criação e transferência de conhecimentos em e entre organizações.

De modo geral, observa-se um consenso de que conhecimento refere-se ao recurso ou ativo aprendido por meio de processos individuais e coletivos de troca e análise de experiências vividas pela própria organização, bem como de experiências de outras organizações (Argote, 2005; Starbuck; Hedberg, 2001; Levitt; March, 1988). A partir das contribuições da Sociologia para o estudo de conhecimento e aprendizagem em organizações, evidencia-se a noção de redes em que, diante de incertezas e rapidez com que conhecimentos e tecnologias são gerados, tornase imprescindível a busca desses recursos em contextos de cooperação organizacional ou de interações sociais formais e informais, conforme destacado por Argote (2005), Reagans e McEvily (2003), Starbuck e Hedberg (2001), Araújo (1998), Miner e Mezias (1996), Lave e Wenger (1991), entre outros autores.

Em decorrência das contribuições da Psicologia, Sociologia e Economia, o termo conhecimento tem sido descrito na literatura de estudos organizacionais como recurso oriundo de indivíduos e interações sociais (De Long; Foley, 2000; Araújo, 1998) que é aplicado em rotinas e

Revista de Administração e Inovação, São Paulo, v. 7, n. 2, p. 127-149, abr./jun. 2010 
capacidades organizacionais (Argote; 2005; Eisenhardt; Martin, 2000; Teece; Pisano; Shuen, 1997; Pisano, 1994) para a solução de problemas (Simon, 1979; March; Simon, 1981; Spender; Grant, 1996) e criação de significado compartilhado entre indivíduos (Gherardi; Nicolini, 2003; Nooteboom, 2001; Nonaka; Takeuchi, 1997).

Além das dimensões tácita e explícita apresentada por Polanyi (1958), De Long e Fahey (2000) destacam o conhecimento estruturado presente em rotinas, processos e sistemas como conhecimento explícito, baseado em regras e independente dos indivíduos por se tornar um recurso da organização. Esse último tipo de conhecimento é associado à noção de aprendizagem em organizações e capacidades dinâmicas (Argote; 2005; Eisenhardt; Martin, 2000; Teece; Pisano; Shuen, 1997; Pisano, 1994), conseqüentemente, à inovação.

De acordo com Argote, McEvily e Reagans (2003), a compreensão do conhecimento em organizações depende de uma integração com a literatura da aprendizagem, conforme também destacam Chiva e Alegre (2005), Argote (2005) e Gherardi e Nicolini (2003). O termo aprendizagem em organizações tem sido comumente descrito como processo de mudança (em rotinas, processos e procedimentos) e Adaptação (vera; crossan, 2005; easterby-smith, 1997; argyris; schön, 1996; march; simon, 1981) por meio de aquisição e desenvolvimento de conhecimentos (nooteboom, 2001; miller, 1996; huber, 1991) que resulta na solução de problemas (fiol; lyles, 1985; simon, 1979) e na criação de significados compartilhados entre indivíduos em organizações (Shirivastava, 1983; Simon, 1979; Cangelosi; Dill, 1965).

Em adição, a aprendizagem tem se referido a um processo multinível (Zietsma Et Al, 2002; Easterby-Smith; Crossan; Nicolini, 2000; Crossan; Lane; White, 1999; Miner; Mezias, 1996) que perpassa indivíduos, grupos e organizações em busca de conhecimentos relevantes que sustentem diferenciais competitivos e solução de problemas. Segundo Miner e Mezias (1996) e March e Simon (1981), a aprendizagem pode resultar em inovações a partir de intenções ou motivações como grau de aspiração por novos patamares de desempenho, modificações no ambiente que tornam os processos organizacionais insatisfatórios e tensão entre aspirações e realizações, por exemplo. Por outro lado, Argyris e Schön (1996), Mezias e Miner (1996) e March (1991) destacam que a aprendizagem não assume caráter positivo ou negativo, mas representa um fenômeno em contextos particulares.

Uma das abordagens da aprendizagem proveniente da Economia sugere que organizações aprendem fazendo (ou pela experiência) e pela interação A idéia de aprender fazendo surge com o trabalho seminal de Arrow (1962) que propõe a aprendizagem como um

Revista de Administração e Inovação, São Paulo, v. 7, n. 2, p. 127-149, abr./jun. 2010 
produto da experiência, sendo observada somente durante a ação de solucionar problemas em organizações, e por repetição de ações bem-sucedidas frente a estímulos contextuais conhecidos. Nessa linha de pensamento, Pisano (1994) afirma que a premissa subjacente ao aprender fazendo refere-se ao fato de que somente a experiência de produção pode fazer com que uma organização descubra problemas que explicam as causas de diferenças entre desempenho atual e desempenho potencial. Ou seja, esses autores mostram que aprendizagem e inovação são conceitos indissociáveis enquanto mecanismos de solução de problemas.

O aprender fazendo está relacionado à noção de capacidades dinâmicas, as quais Teece, Pisano e Shuen (1997), e Eisenhardt e Martin (2000) definem como a habilidade de uma organização integrar, construir e reconfigurar competências organizacionais internas e externas para responder às mudanças contextuais. Essa definição remete à noção de rotinas, na visão evolucionária de Nelson e Winter (2005), como todos os padrões comportamentais regulares e previsíveis de organizações que regulam suas ações ao longo do tempo e são historicamente construídos a partir da experiência.

Outra abordagem da aprendizagem na perspectiva econômica é a denominada "Economia do Aprendizado" em que Lundvall e Johnson (1994) destacam o papel primordial do conhecimento e da aprendizagem na mudança e crescimento econômico. Segundo esses autores, mudanças técnicas e econômicas ocorrem a partir de novas combinações de conhecimentos aplicadas ao um novo know-how que permite novas formas de produção, difusão e entrega de bens e serviços, o que permite inferir um resgate da "economia de trocas" de Schumpeter (1985).

Lundvall e Johnson (1994) preconizam a noção de aprendizagem pela interação para destacar novas formas de aquisição de conhecimentos diante de mudanças importantes na economia global, principalmente aquelas impactadas pelas tecnologias de informação e comunicação (TIC's). Entretanto, esses autores destacam que as TIC's não reduziram os custos de aquisição de informações e conhecimentos, o que determinou a necessidade de organizações buscarem esses recursos em fontes externas por meio de redes de cooperação e alianças estratégicas.

Complementando as proposições de Lundvall e Johnson (1994), Lundvall (1996) mostra que o crescimento de redes de conhecimento entre organizações se deu em virtude da complexidade das bases de conhecimento e de mudanças contextuais cada vez mais rápidas, motivando relações seletivas e de longo prazo para a produção e distribuição de conhecimentos. Segundo Lundvall e Johnson (1994), o "saber o que" e o "saber por que" incluem diferentes 
elementos de informações triviais e têm características comuns, tais como: (a) referem-se a conhecimentos explícitos, passíveis de descrição e reprodução por outros; (b) representam commodities; (c) altos custos de transação. O "saber quem, quando e onde" e o "saber como" diferem-se e representam uma dimensão mais tácita ou intangível do conhecimento. Nessa linha, o crescimento econômico seria determinado por novas combinações de novos métodos de produção e por novos produtos, o que significa dizer que o saber como fazer e mudar as coisas é mais difícil do que aprender.

O saber quem é descrito por Lundvall e Johnson (1994, p. 29) com a seguinte frase "eu lhe apresento essa importante pessoa e depois você me coloca em contato com seus amigos mais influentes". Para esses autores, a combinação dessas categorias de conhecimento configura a inovação e a aprendizagem pela interação, conforme também destacam Miner e Mezias (1996) e Starbuck e Hedberg (2001) ao se referirem à aprendizagem na perspectiva da população de organizações.

Em decorrência das idéias construídas até o momento, infere-se que na "Economia do Aprendizado" vislumbra-se também o aporte teórico necessário para a articulação entre conhecimento, aprendizagem e inovação em organizações. Em outras palavras, o conhecimento refere-se ao recurso economicamente valioso e sua dimensão mais tácita e inimitável configurase como a essência da dinâmica organizacional (Vargas; Zawislak, 2006). Além disso, a aprendizagem por meio de relações entre indivíduos e organizações representa de que maneira conhecimentos podem ser combinados para resultar em inovações e em mudanças técnicas e econômicas. Dessa forma, se chega às respostas para as duas questões essenciais sobre conhecimento e aprendizagem: o que é aprendido e como é aprendido. Para cumprir o objetivo de articular teoricamente conhecimento, aprendizagem e inovação em organizações, a seguir será apresentada a construção teórica sobre inovação.

\subsection{UMA VISÃO GERAL SOBRE INOVAÇÃO}

A inovação é um dos fenômenos que sempre esteve presente na história da humanidade, assim como o conhecimento e a aprendizagem. Apesar de sua óbvia importância para a sociedade, esse tema nem sempre recebeu a atenção que merece (Fagerberg, 2005). Por muito tempo, a Economia reinou como principal área do conhecimento a estudar esse fenômeno, concentrando-se em identificar incentivos econômicos para a inovação, bem como seus efeitos para mercados e países (Pavitt, 2005).

Revista de Administração e Inovação, São Paulo, v. 7, n. 2, p. 127-149, abr./jun. 2010 
Os estudos sobre inovação, principalmente na Economia, têm seu marco inicial na década de 1930 com a publicação do livro The Theory of Economic Development de autoria de Joseph Alois Schumpeter. Em sua proposta, Schumpeter (1985) apresenta a inovação como "novas combinações", isto é, fenômeno fundamental do desenvolvimento econômico. Esse autor afirma que a combinação de recursos disponíveis pode resultar em algo diferente ou apenas modificar a forma como fazê-lo. Nesse sentido, observa-se a predominância de duas ênfases sobre inovação influenciadas por Schumpeter (1985): produto tecnológico e produção tecnológica (Fargerberg, 2005), resultando nos termos inovação de produto e inovação de processo, respectivamente. Aquele autor apresenta ainda outros tipos de inovação: abertura de um novo mercado, aquisição de uma nova fonte de oferta de matéria-prima e o surgimento de uma nova organização de qualquer indústria.

O desenvolvimento do campo da inovação apoiou-se nas críticas de pesquisadores à Teoria do Comportamento da Firma preconizada por Cyert e March em 1963. De acordo com Nelson e Winter (2005), essa teoria lança luz sobre os fenômenos econômicos de mercado como previsíveis e de imediato ajustamento comportamental por parte de firmas, e por isso torna-se frágil para explicar a complexidade e dinamismo da inovação. Schumpeter (1985) e seus seguidores Nelson e Winter (2005) são considerados pilares importantes da Economia Evolucionária que enfatiza a "importância da variedade e da diversidade tecnológicas e das formas em que a variedade se traduz em oportunidades e resultados tecnológicos" (OCDE, 1997, p.35). Tais oportunidades impactam a capacidade de inovação de organizações e suas trajetórias.

O conceito de inovação se difundiu a partir das contribuições de Schumpeter e na década de 1960 emergiu como um campo próprio de pesquisa, e tem sido comumente associado a outros fenômenos em diferentes níveis. No nível individual, fenômenos como criatividade (Amabile Et Al, 1996; Gurteen, 1998), cognição e decisão (March; Simon, 1981; Daft; Weick, 1984), liderança, background acadêmico (Kimberly; Evanisko, 1981) estão associados à inovação, com destaque para a perspectiva psicológica da inovação. No nível organizacional, a inovação é associada à capacidade de absorção de conhecimentos (Cohen; Levinthal, 1990; Tsai, 2001), com investimentos em pesquisa e desenvolvimento (Kim, 2005; Mowery; Rosenberg, 2005), conhecimento e aprendizagem (Nelson; Winter, 2005; Daft; Weick, 1984; Cohen; Levinthal, 1990; Lundvall; Johnson, 1994), adoção tecnológica (Kimberly; Evanisko, 1981; Damanpour, 1991; Pérez Et Al, 2004) e capacidades dinâmicas (Teece; Pisano; Shuen, 1997; Eisenhardt; Martin, 2000). No nível da indústria e países, a inovação também está relacionada com políticas científica e tecnológica e sistemas de inovação (Malerba, 2003; Lundvall; Borrás, 2005; Dodgson, 2005).

Revista de Administração e Inovação, São Paulo, v. 7, n. 2, p. 127-149, abr./jun. 2010 
Um ponto importante da literatura sobre inovação refere-se à trajetória de estudos empreendidos nessa área. A primeira vertente refere-se à inovação enquanto processo de difusão e adoção de novas tecnologias e abarca estudos descritivos em termos de características ou padrões de adoção de inovações no tempo e espaço, tais como atributos de adotantes, redes sociais em que adotantes atuam, atributos da inovação, características ambientais, comunicação de inovações e características de quem promove inovações (Downs; Mohr, 1976; Bigoness; Perreault, 1981; Kimberly; Evanisko, 1981; Wolfe, 1994).

A segunda vertente caracteriza-se em compreender inovação na perspectiva de seus determinantes organizacionais, isto é, atributos organizacionais que favorecem ou inibem seu desenvolvimento. Nessa vertente, os estudos recaem sobre inovatividade e descrevem antecedentes como o número de inovações adotadas e estrutura (Damanpour, 1991; Wolfe, 1994), investimentos em pesquisa e Desenvolvimento (Nelson; Winter, 2005; Mowery; RosenberG; 2005), tamanho da organização, especialização de mão-de-obra, variedade de unidades organizacionais, integração com o ambiente externo (Kimberly; Evanisko, 1981).

A terceira vertente de estudos aborda a inovação na perspectiva de processo, sobretudo suas fases ou estágios. Procura-se desenvolver uma teoria que descreve a natureza do processo de inovação com ênfase no como e por que inovações surgem, desenvolvem-se, crescem e terminam. Essa corrente de pesquisa segue duas direções: (a) modelo de estágios da inovação; (b) estudos longitudinais e em profundidade sobre inovação. Os estudos baseados na perspectiva de estágios ou fases consideram a inovação como um processo que perpassa pela decisão por uma inovação (aware), identificação de oportunidades (match), avaliação de custos e benefícios (appraise), adoção ou rejeição, implementação, confirmação ou refutação, e aceitação de uma inovação como rotina organizacional (Wolfe, 1994; Daft, 1978).

Segundo Steiner (1995), a inovação assume um caráter complexo e dinâmico apoiado na natureza incerta e não-convencional de indivíduos e organizações, bem como de suas interações num ambiente natural. Em outras palavras, a inovação revela-se como um fenômeno de natureza interacional e cambiante em virtude de diversas forças contextuais (Freeman, 1991; Schumpeter, 1985). À luz das idéias seminais de Arrow (1962) e Schumpeter (1985), Lundvall e Johnson (1994) se propõem a entender a dinâmica e complexidade da inovação na perspectiva da "Economia do Aprendizado", conforme destacado na seção anterior. Esses autores reafirmam a noção da interação como variável explicativa de mudanças técnicas e econômicas significativas, postulada anteriormente por Schumpeter (1985) ao se referir à “economia de trocas".

Revista de Administração e Inovação, São Paulo, v. 7, n. 2, p. 127-149, abr./jun. 2010 
Segundo Lundvall e Johnson (1994), na Economia do Aprendizado, organizações buscam novas formas de aprender a partir de novos conhecimentos utilizados na produção (de novos produtos ou novos processos). A mudança técnica é tida como uma renda privilegiada (rent-seeking) em tempos de competição, e, diante disso, organizações sempre procuram algum grau de reconhecimento no mercado, seja por meio de combinações de habilidades, conhecimentos e experiências de indivíduos atuantes em pesquisa e desenvolvimento, seja perseguindo trajetórias tecnológicas específicas da indústria.

Na verdade, Lundvall e Johnson (1994) evidenciam que mudança tecnológica requer trocas constantes de informações e conhecimentos entre diferentes pessoas em diferentes unidades organizacionais e em diferentes níveis na perspectiva intra e interorganizacional, visto que as novas tecnologias de informação e comunicação não reduzem os custos de aquisição de informações e conhecimentos, determinando a necessidade de organizações buscarem esses recursos em fontes externas por meio de redes de cooperação e alianças estratégicas. Diante disso, a inovação em organizações tem sido estudada, nos últimos anos, na perspectiva de redes assim como conhecimento e aprendizagem em organizações.

Segundo Pellegrin et al (2007), a noção de redes de inovação é sombreada pelo conceito de sistemas de inovação. Esses autores afirmam que as redes de inovação envolvem processos de interação entre atores sociais distintos, produzindo inovações em diversos níveis de agregação, tais como regional, nacional e global. Staropoli (1998) mostra que a cooperação entre organizações depende de trocas humanas, e de recursos tecnológicos e financeiros entre diferentes e independentes atores sociais que freqüentemente competem entre si. A dimensão das redes em estudos de inovação também é apresentada por Vargas e Zawislak (2006) como determinante do processo de aprendizagem, do ritmo e da direção de inovações, resultando na evolução do sistema como um todo.

$\mathrm{Na}$ seção seguinte procura-se integrar teoricamente conhecimento, aprendizagem e inovações em organizações considerando suas peculiaridades, mas ressaltando os aspectos comuns aos três campos de estudos. A seguir serão apresentadas as diferenças e similaridades encontradas a partir da definição dos construtos e características do cada campo. Em adição, será abordada proposta de integração teórica com indicação das proposições que orientarão as pesquisas relativas a essa idéia.

Revista de Administração e Inovação, São Paulo, v. 7, n. 2, p. 127-149, abr./jun. 2010 


\section{ARTICULAÇÃO TEÓRICA ENTRE CONHECIMENTO, APRENDIZAGEM E INOVAÇÃO EM ORGANIZAÇÕES}

Os campos do conhecimento, aprendizagem e inovação em organizações apresentam interfaces, similaridades e diferenças teórico-conceituais, como proposto na Figura 1. As similaridades e diferenças entre conhecimento e aprendizagem em organizações são baseadas em Cook e Brown (1999), Vera e Crossan (2005) e Chiva e Alegre (2005). A inserção da inovação é feita com base em revisão de literatura apresentada na seção anterior.

Abordagem Descritiva:

Abordagem Descritiva:

Conhecimento em Organizações Aprendizagem em Organizações

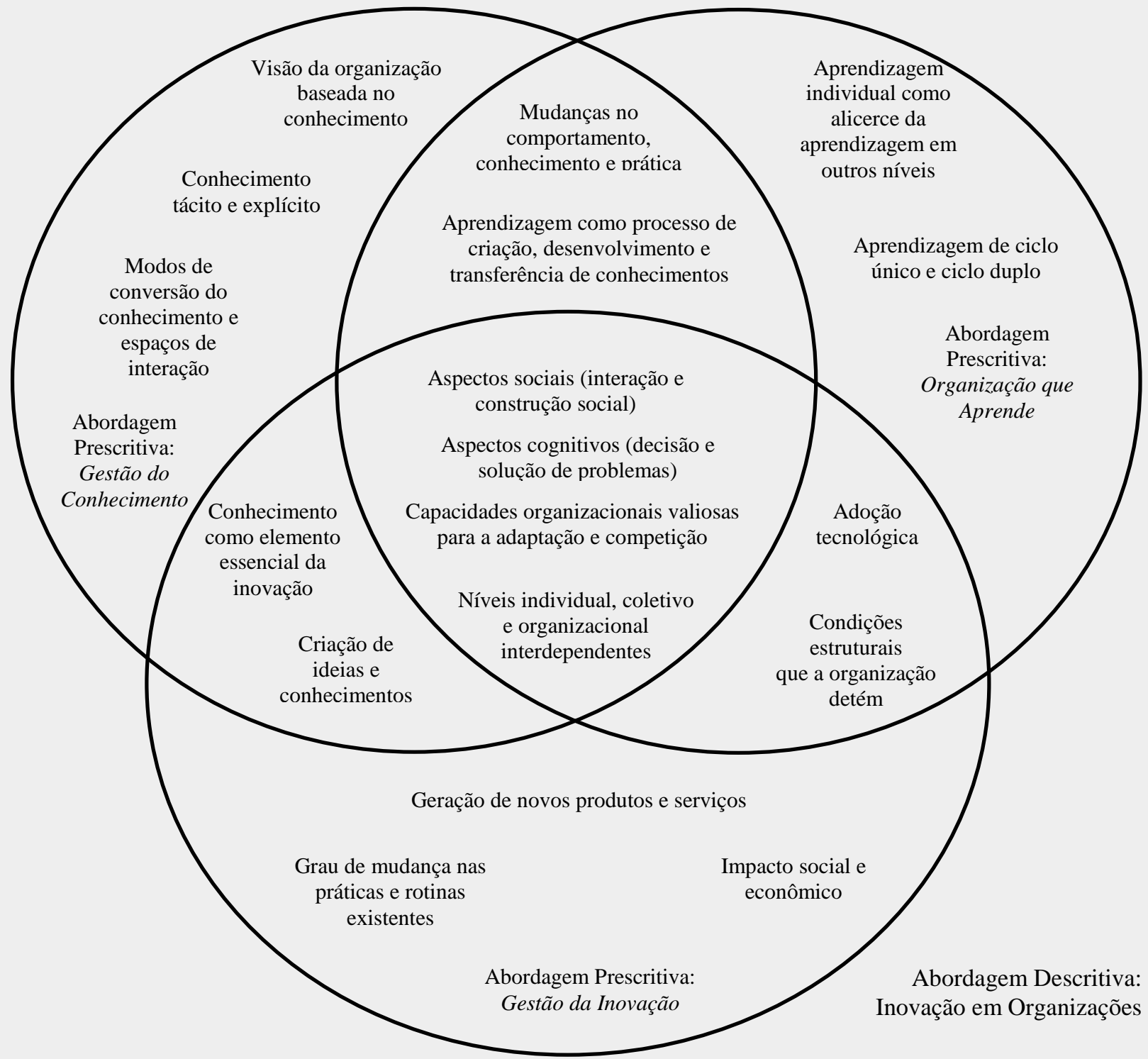

Figura 1: Interfaces e limites entre conhecimento, aprendizagem e inovação em organizações.

Fonte: elaboração dos autores. 
O marco conceitual descrito em seções anteriores e a Figura 1 sugerem que conhecimento, aprendizagem e inovação em organizações são caracterizados por diversidade conceitual. As três áreas são similares em termos ontológicos e epistemológicos quanto às principais perspectivas de estudo mais citadas. Essas abordagens não são excludentes, mas enfatizam aspectos diferentes desses fenômenos em contextos organizacionais. A abordagem cognitiva prioriza variáveis individuais como, por exemplo, tomada de decisão, solução de problemas, criatividade, informações, valores, entre outras, enquanto que as abordagens social e econômica ressaltam esses fenômenos enquanto processos sociais de construção, transformação e aplicação de algo considerado relevante para o contexto ou mercado. Em outras palavras, conhecimento, aprendizagem e inovação envolvem a interação de diversos atores sociais organizacionais e podem ser facilitados ou inibidos também por variáveis contextuais, estruturais, e sociais e individuais.

Os três campos também convergem quanto aos principais níveis em que esses fenômenos ocorrem. Percebe-se que são estudados nos níveis individual, coletivo, organizacional, interorganizacional e societal, e são interdependentes. Há convergência também quando são considerados diferenciais para as organizações se adaptarem, competirem e se diferenciarem em relação ao mercado e seus concorrentes, resultando em ganhos de desempenho. Outra similaridade refere-se ao fato de os três campos terem, além de abordagens descritivas, abordagens prescritivas, ou seja, tentativas de valoração positiva do conceito, ênfase em intervenção e prescrições de ordem prática. Essas perspectivas merecem atenção de acadêmicos e práticos, pois podem levar a atitudes acríticas frente a circunstâncias que exijam um posicionamento reflexivo acerca da realidade organizacional.

Vera e Crossan (2005) fazem duas proposições para integração dos campos de conhecimento e aprendizagem em organizações. A primeira propõe uma relação de feedback em que o processo de aprendizagem produz novos conhecimentos no contexto organizacional. A segunda afirma que as estratégias organizacionais voltadas para conhecimento e aprendizagem e as estratégias de negócio, quando alinhadas, moderam positivamente a relação entre conhecimento, aprendizagem e desempenho. A partir das idéias dessas autoras, neste texto insere-se a inovação como componente da relação entre conhecimento e aprendizagem em organizações. Conforme já tratado neste ensaio, a inovação tem sido freqüentemente associada à adoção de novas práticas, tecnologias e produtos. Entretanto, Pérez et al (2004) sugerem que a inovação em contextos organizacionais depende também da intenção em adotar algo considerado novo na organização. Assim, a partir da intenção em inovar a organização mobilizará recursos para alcançar resultados positivos de desempenho. 
Sendo assim, a inovação é vista como o resultado de processos de aprendizagem que geram e aplicam novos conhecimentos em rotinas, processos e procedimentos. A mensuração desse resultado permite identificar o impacto (social e/ou econômico) da adoção, da criação ou desenvolvimento de algo tido como novo. A noção de resultado sugere que a inovação é contextualizada, ou seja, considera demandas, intenções e necessidades do ambiente interno ou externo à organização.

A idéia central é ressaltar a interface entre esses construtos na perspectiva organizacional, visto que há uma literatura fragmentada em relação a cada conceito. Inicialmente, a noção de conhecimento refere-se ao que é criado, desenvolvido, transferido e aplicado no contexto organizacional. Nesse sentido, a aprendizagem abarca o processo de construção do conhecimento, seja ele individual ou coletivo, e seu resultado pode ou não ser uma inovação. Entretanto, espera-se que inovações sejam geradas, pois, em organizações existem objetivos, demandas, necessidades, interesses que motivam a mudança das práticas vigentes.

Um dos principais aspectos convergentes dos constructos apresentados é a noção de redes ou alianças entre organizações em virtude de limitações de recursos e capacidades organizacionais para o aprendizado e inovação. De outra forma, as relações entre organizações configuram-se como alternativas estratégicas para a vantagem competitiva em tempos de instabilidade (Dyer; Singh, 1998; Powell, 1998; Powell; Koput; Smith-Doerr, 1996; Ring; Van De Ven, 1994). Além disso, é na perspectiva interacional que se entra em contato com o caráter dinâmico e complexo do conhecimento, aprendizagem e inovação em organizações (Lundvall, 1996; Lundvall; Johnson, 1994).

A literatura sobre conhecimento, aprendizagem e inovação, na perspectiva de redes, provém principalmente de estudos apoiados na Psicologia, Sociologia e Economia. Nas duas primeiras disciplinas nota-se a predominância do termo redes sociais para descrever relações estabelecidas entre indivíduos e grupos, bem como a estruturação (atributos e características) de redes em busca de compartilhar conhecimentos e aprendizagem a partir de trocas de experiências (Van Wijk; Jansen; Lyles, 2008; Nahapiet; Ghoshal, 1998). Na Economia percebe-se a predominância de termos como alianças estratégicas, joint ventures, redes de cooperação, redes intra e interorganizacionais para descrever relações estabelecidas entre organizações em espaços institucionais visando aquisição e compartilhamento de recursos e capacidades que signifiquem diferenciais competitivos (Dyer; Singh, 1998; Powell, 1998; Powell; Koput; Smith-Doerr, 1996; Gulati, 1995; Ring; Van de Ven, 1994; Powell, 1987).

Revista de Administração e Inovação, São Paulo, v. 7, n. 2, p. 127-149, abr./jun. 2010 
Com base na constatação da fragmentação conceitual presente na literatura de estudos organizacionais acerca de conhecimento, aprendizagem e inovação em organizações, infere-se a existência de uma lacuna teórica e metodológica representada na seguinte questão: como é possível compreender a dinâmica e complexidade inerentes à mudança técnica separando a dimensão social e econômica desses fenômenos? A resposta parece estar situada no "Paradigma Tecno-Econômico" apresentado com destaque por Freeman (1991) e DeBresson e Amesse (1991).

Com a prerrogativa de adquirir informações para a geração de conhecimentos valiosos para a inovação, Freeman (1991) e DeBresson e Amesse (1991) afirmam que as redes atuam como catalisadoras de acesso e transformação de recursos em produtos e serviços inovadores. Essa ênfase ganha notoriedade a partir da década de 1980, principalmente, com a importância dada à dimensão tácita do conhecimento que somente é acessada por meio de interações muitas vezes informais. Além disso, a noção de redes justifica-se pela forte incerteza tecnológica e de mercado que caracteriza as décadas de 1980 e 1990 (Debresson; Amesse, 1991). Sendo assim, tem-se o terreno fértil para uma nova proposta de compreensão da mudança técnica.

A partir da década de 1990, a noção de cooperação entre organizações para a pesquisa e desenvolvimento é influenciada pelo desenvolvimento de tecnologias de informação e comunicação que reduziram o tempo entre adoção e ganho de mercado por meio da diminuição dos custos de compartilhamento de recursos e do desenvolvimento de tecnologias complementares (Freeman, 1991; Debresson; Amesse, 1991). Percebe-se, então, que a limitação do acesso a conhecimentos relevantes (Wolfe, 1994) para a inovação é atenuada com a incorporação de tecnologias que incrementam a comunicação entre atores de redes e potencializam a difusão de inovações para novos setores. Em síntese, o paradigma tecnoeconômico caracteriza-se pela intensificação da colaboração tecnológica entre organizações e seus fornecedores, entre consumidores e produtores de tecnologias de informação e comunicação, bem como pela acirrada competição entre fornecedores daqueles que produzem tecnologias (Freeman, 1991). A dinâmica e complexidade da inovação estão exatamente nessas interações colaborativas e competitivas (Debresson; Amesse, 1991).

Outro aspecto que merece destaque para responder à questão apresentada anteriormente refere-se a noção de "Redes Tecno-Econômicas". Segundo Green et al (1999), as redes tecnoeconômicas são a representação ideal da integração entre a dimensão social e a dimensão econômica da inovação. De acordo com Callon (2007), essas redes representam formas de arranjo de mercado que são constituídas de atores heterogêneos coordenados e descrevem 
relações fortes entre ciência, tecnologia e mercado. Dito de outra forma, tais redes procuram mostrar como organizações interagem numa malha de transações cooperativas que potencializam a inovação e os ganhos de competitividade.

Para Callon (2007), as redes tecno-econômicas requerem a singularização, socialização, integração, reação e adaptação por parte de grupos de atores diversificados e heterogêneos num contexto de mercado ou numa economia. Essas redes podem ser (re)configuradas dependendo de demandas de alianças estratégicas, desenho de inovações e necessidades de consumidores. Além disso, novas relações e formas de organização podem ocorrer em virtude de interações dinâmicas entre organizações, acentuando a influência do individualismo na vida econômica. É com base nessas idéias que se apóia a noção de dinâmica e complexidade da inovação.

Com base nas proposições de Callon (2007), é importante destacar de que forma conhecimento, aprendizagem e inovação em organizações podem ser integrados na perspectiva de redes tecno-econômicas. A inovação representa algo novo que emerge da (re)combinação de recursos no sentido schumpeteriano. O novo, então, representa a característica tácita do conhecimento subjacente à inovação (Dosi, 1988), isto é, a dimensão mais intrínseca de um ativo determinante para a mudança técnica (Lundvall, 1996; Lundvall; Johnson, 1994). Para o novo ser aceito e reconhecido socialmente, este precisa ser comunicado apropriadamente, bem como produzido de forma integrada para que faça sentido num contexto específico (Wolfe, 1994; Freeman, 1991; Debresson; Amesse, 1991). Dessa forma, a socialização do novo se dá a partir do aprendizado pela interação (Lundvall; Johnson, 1994) que permite conhecer as necessidades de futuros usuários de novas tecnologias (Freeman, 1991), isto é, a singularização do novo (CALLON, 2007).

Entretanto, para que o novo seja produzido de modo efetivo, diversos tipos de conhecimentos são combinados, especialmente conhecimentos estruturados (De Long; Foley, 2000) em rotinas e procedimentos organizacionais (Nelson; Winter, 2005; Eisenhardt; Martin, 2000; Teece; Pisano; Shuen, 1997) para a concepção, produção e distribuição de inovações, isto é, a coletividade integrada (Callon, 2007). Por outro lado, essa coletividade integrada depende de interações entre diferentes atores (heterogêneos) para o acesso e compartilhamento de recursos e capacidades organizacionais necessárias à inovação (Debresson; Amesse, 1991), o que só é possível a partir do conhecimento de quem, quando e onde procurar e estabelecer relações com outros atores que detêm recursos valiosos para a inovação (Lundvall; Johnson, 1994). Em síntese, a inovação é um fenômeno dinâmico porque envolve diversas variáveis contraditórias (Callon, 2007), representando um oximoro entre aprendizagem e organização (Weick; Westley,

Revista de Administração e Inovação, São Paulo, v. 7, n. 2, p. 127-149, abr./jun. 2010 
1996), entre cooperação e competição (Freeman, 1991), entre organizar e inovar. Além disso, inovar é complexo, pois há uma variedade de atores heterogêneos que interagem de modo coordenado em busca de ganhos de competitividade.

O objetivo dessa seção foi articular teoricamente conhecimento, aprendizagem e inovação em organizações. Para alcançar esse objetivo, buscou-se no paradigma da "Economia do Aprendizado" e no paradigma "Tecno-Econômico" os argumentos teóricos que sustentam a proposta de integração apresentada. Como produto final dessa articulação o termo conhecimento organizacional é definido como "ativo ou recurso, oriundo de indivíduos e interações sociais, incrustado em rotinas e capacidades organizacionais, para a produção de bens e serviços e criação de significado compartilhado entre indivíduos". O termo aprendizagem organizacional é definido como "processo multinível de interação entre indivíduos e grupos, com troca de conhecimentos que resultam em mudança e adaptação organizacional". O termo inovação, apoiando-se em uma das concepções de Schumpeter (1985) que a define como "novas combinações de recursos", é concebido como "o resultado de processos de aprendizagem em que conhecimentos relevantes são combinados e estruturados em novas soluções e significados compartilhados".

\section{CONSIDERAÇÕES FINAIS}

O presente artigo realizou uma discussão articulada a respeito dos conceitos de conhecimento, aprendizagem e inovação em organizações, com ênfase para a polissemia e o caráter multifacetado com que esses termos são tratados em distintos campos do conhecimento. Tratou, também, de distinções importantes nesses campos, como as diferenças entre conhecimento em organizações e gestão do conhecimento, entre aprendizagem em organizações e organização que aprende, e entre inovação em organizações e gestão da inovação. Em relação às pesquisas que considerem as propostas aqui empreendidas, é importante ressaltar que a utilização de métodos qualitativos e quantitativos de maneira combinada nas pesquisas favorece uma compreensão mais segura dos fenômenos aqui discutidos.

Estudos futuros poderiam enfatizar inovações organizacionais implementadas e reconhecidas, descrever o processo de aprendizagem ocorrido e identificar os conhecimentos gerados, transferidos e aplicados nas práticas organizacionais. Pesquisas também poderiam explorar o caráter longitudinal evidenciando os conhecimentos identificados como relevantes para organizações, com o intuito de descrever todo o processo, desde a identificação do conhecimento até seus resultados efetivos para uma organização.

Revista de Administração e Inovação, São Paulo, v. 7, n. 2, p. 127-149, abr./jun. 2010 
Em se tratando de inovação, descrever os impactos sociais e/ou econômicos advindos de sua geração e adoção é uma alternativa de pesquisa. Isso indica estudos de ênfase quantitativa que podem descrever os impactos dos resultados (econômicos e financeiros, por exemplo) de inovações no desempenho organizacional; descrever os efeitos dos investimentos em processos de aprendizagem no desempenho organizacional durante sua ocorrência, ou seja, testar as hipóteses de exploration e exploitation propostas por March (1991), por exemplo. Para concluir, este ensaio pretendeu oferecer um mapa preliminar de como conhecimento, aprendizagem e inovação em organizações se relacionam. De fato, há muito que ser aprendido sobre esses fenômenos. Espera-se que estudos futuros ampliem as bases teóricas que sustentem a integração proposta e desenhem novas pesquisas para o avanço desse campo convergente como um todo.

\section{REFERÊNCIAS}

Amabile, Teresa M.; Conti, Regina; Coon, Heather; Lazenby, Jeffrey; Herron, Michael. Assessing the work environment for creativity. Academy of Management Journal, vol. 39, n. 5, p. 1154-1184, 1996.

Araújo, Luis. Knowing and learning as networking. Management Science, vol. 29, n. 3, p. 317336, 1998.

Argote, Linda. Reflections on two views of managing learning and knowledge in organizations. Journal of Management Inquiry, vol. 14, n. 1, p. 43-48, 2005.

Argote, Linda; Ingram, Paul; LEVINE, John M.; MORELAND, Richard L. Knowledge transfer in organizations: learning experience of others. Organizational Behavior and Human Decision Process, vol. 82, n. 1, p. 1-8, 2000.

Argote, Linda; Mcevily, Bill; Reagans, Ray. Management knowledge in organizations: a integrative framework and review of emerging themes. Management Science, vol. 49, n. 4, p. 571-582, 2003.

Argyris, Chris; Schön, Donald A. Organizational learning II: theory, method, and practice. Reading, MA: Addison-Wesley, 1996.

Arrow, Kenneth J. The economic implications of learning by doing. The Review of Economic Studies, vol. 29, n. 3, p. 155-173, 1962.

Balestrin, Alsones; Vargas, Lilia Maria; Fayard, Pierre. Knowledge creation in small-firm network. Journal of Knowledge Management, vol. 12, n. 2, p. 94-106, 2008.

Bigoness, William J.; Perreault, William D. A conceptual paradigm and approach for the study of innovators. Academy of Management Review, vol. 24, n. 1, p. 68-82, 1981.

Revista de Administração e Inovação, São Paulo, v. 7, n. 2, p. 127-149, abr./jun. 2010 
Borgatti, Stephen P.; Foster, Pacey C. The network paradigm in organizational research: a review and typology. Journal of Management, vol. 29, n. 6, p. 991-1013, 2003.

Callon, Michel. An essay on the growing contribution of economic markets to the proliferation of the social. Theory, Culture \& Society, vol. 24, n. 7/8, p. 139-163, 2007.

Cangelosi, Vincent E.; DILL, William R. Organizational learning: observations toward a theory. Administrative Science Quarterly, vol. 10, n. 2, p. 175-203, 1965.

Chiva, Ricardo; Alegre, Joaquín. Organizational learning and organizational knowledge: towards the integration of two approaches. Management Learning, vol. 36, n. 1, p. 49-68, 2005.

Cohen, Wesley M.; Levinthal, Daniel A. Absorptive capacity: a new perspective on learning and innovation. Administrative Science Quarterly, vol. 35, n. 1, p.128-152, 1990.

Cook, Scott D. N.; Brown, John Seely. Bridging epistemologies: the generative dance between organizational knowledge and organizational knowing. Organization Science, vol. 10, n. 4, p. 381-400, 1999.

Crossan, Mary M.; Lane, Henry W.; White, Roderick E. An organizational learning framework: from intuition to institution. The Academy of Management Review, vol. 24, n. 3, p. 522-537, 1999.

Daft, Richard L. A dual-core model of organizational innovation. Academy of Management Journal, vol. 21, n. 2, p. 193-210, 1978.

Daft, Richard L.; Weick, Karl E. Toward a model of organizations as interpretation systems. Academy of Management Review, vol. 9, n. 2, p. 284-295, 1984.

Damanpour, Fariborz. Organizational innovation: a meta-analysis of effects of determinants and moderators. Academy of Management Journal, vol. 34, n. 3, p. 555-590, 1991.

De Long, David W.; Fahey, Liam. Diagnosing cultural barriers to knowledge management. Academy of Management Executive, vol. 14, n. 4, p. 113-127, 2000.

Debresson, Chris; Amesse, Fernand. Networks of innovators: a review and introduction to the issue. Research Policy, vol. 20, p. 363-379, 1991.

Dodgson, Mark. As políticas para ciência, tecnologia e inovação nas empresas asiáticas de industrialização recente. In: Linsu Kim e Richard R. Nelson (Orgs.). Tecnologia, aprendizado e inovação: experiências das economias de industrialização recente. Campinas-SP: Editora da UNICAMP, p. 313-364, 2005.

Dosi, Giovanni. Sources, procedures, microeconomic effects of innovation. Journal of Economic Literature, vol. 26, p. 1120-1171, 1988.

Downs, George W.; Mohr, Laurence B. Conceptual issues in the study of innovations. Administrative Science Quarterly, vol. 21, p. 700-714, 1976.

Revista de Administração e Inovação, São Paulo, v. 7, n. 2, p. 127-149, abr./jun. 2010 
Dyer, Jeffrey H.; Singh, Harbir. The relational view: cooperative strategy and sources of interorganizational competitive advantage. Academy of Management Review, vol. 23, n. 4, p. 660-679, 1998.

Easterby-Smith, Mark. Disciplines of organizational learning: contributions e critiques. Human Relations, vol. 50, n. 9, p. 1085-1113, 1997.

Easterby-Smith, Mark; Crossan, Mary; Nicolini, David. Organizational learning: debates past, present and future. Journal of Management Studies, vol. 37, n. 6, p. 783-796, 2000.

Eisenhardt, Kathleen M.; Martin, Jeffrey A. Dynamic capabilities: what are they? Strategic Management Journal, vol. 21, n. 10/11, p. 1105-1121, 2000.

Elkjaer, Bente. Social learning theory: learning as participation in social process. In: Mark Easterby-Smith e Marjorie A. Lyles (Eds.). Handbook of organizational learning and knowledge management. Oxford: Blackwell Publishing, p. 38-53, 2005.

Fagerberg, Jan. Innovation: a guide to the literature. In: Jan Fagerberg, David C. Mowery e Richard R. Nelson (Eds.). The Oxford Handbook of Innovation. Oxford: Oxford University Press, p. 1-27, 2005.

Fiol, C. Marlene; Lyles, Marjorie A. Organizational learning. The Academy of Management Review, vol. 10, n. 4, p. 803-813, 1985.

Freeman, Christopher. Innovation, changes of techno-economic paradigm and biological analogies in economics. Revue Économique, vol. 42, n. 2, p. 211-231, 1991.

Gherardi, Silvia; Nicolini, Davide. The Sociological foundations of organizational learning. In: Meinolf Dierkes, Ariane Berthoin Antal, John Child e Ikujiro Nonaka. Handbook of organizational learning and knowledge. Oxford: Oxford University Press, p. 35-60, 2003.

Grant, Robert M. Toward a knowledge-based theory of the firm. Strategic Management Journal, vol. 17, p. 109-122, 1996.

Green, Ken; Hull, Richard; Mcmeekin, Andrew; Walsh, Vivien. The construction of the technoeconomic: networks vs. paradigms. Research Policy, vol. 28, p. 777-792, 1999.

Gulati, Ranjay. Social structure of alliance formation patterns: a longitudinal analysis. Administrative Science Quarterly, vol. 40, p. 619-652, 1995.

Gurteen, David. Knowledge, creativity and innovation. Journal of Knowledge Management, vol. 2, n. 1, p. 5-13, 1998.

Hargadon, Andrew B. Brokering knowledge:linking learning and innovation. Research in Organizational Behavior, vol. 24, p. 41-85, 2002.

Huber, George P. Organizational learning: the contributing process and the literatures. Organization Science, vol. 2, n. 1, p. 88-115, 1991. 
Kim, Linsu. Da imitação à inovação: a dinâmica do aprendizado tecnológico da Coréia. Campinas-SP: Editora da UNICAMP, 2005.

Kimberly, John R.; Evanisko, Michael J. Organizational innovation: the influence of individual, organizational, and contextual factors on hospital adoption of technological and administrative innovations. Academy of Management Journal, vol. 24, n. 4, p. 689-713, 1981.

Lave, Jean; Wenger, Etienne. Situated learning: legitimate peripheral participation. Cambridge: Cambridge University Press, 1991.

Levitt, Barbara; March, James G. Organizational learning. Annual Review of Sociology, vol.14, p. 319-340, 1988.

Lundvall, Beng-Ake; Borrás, Susana. Science, techonology, and innovation policy. In: Jan Fagerberg, David C. Mowery e Richard R. Nelson (Eds.). The Oxford Handbook of Innovation. Oxford: Oxford University Press, p. 599-631, 2005.

Lundvall, Bengt-Ake. The social dimension of the learning economy. DRUID Working Paper, n. 96-1, 1996.

Lundvall, Bengt-Ake; Johnson, Björn. The learning economy. Journal of Industry Studies, vol. 1, n. 2, p. 23-42, 1994.

Malerba, Franco. Sectoral systems and innovation and technology policy. Revista Brasileira de Inovação, vol. 2, n. 2, p. 329-375, 2003.

March, James G. Exploration and exploitation in organizational learning. Organization Science, vol. 2, n. 1, special issue, p. 71-87, 1991.

March, James G.; Simon, Herbert A. Teoria das organizações. Rio de Janeiro: FGV, 1981.

Miller, Dany. A preliminary typology of organizational learning: synthesizing the literature. Journal of Management, vol. 22, p. 485-505, 1996.

Miner, Anne S.; Mezias, Stephen J. Ugly duckling no more: pasts and futures of organizational learning research. Organization Science, vol. 7, n. 1, p. 88-99, 1996.

Mowery, David C.; Rosenberg, Nathan. Trajetórias da inovação: a mudança tecnológica dos Estados Unidos da América no século XX. Campinas-SP: Editora da UNICAMP, 2005.

Nahapiet, Janine; Ghoshal, Sumantra. Social capital, intellectual capital, and the organizational advantage. The Academy of Management Review, vol. 23, n. 2, p. 242-266, 1998.

Nelson, Richard R.; Winter, Sidney G. Uma teoria evolucionária da mudança econômica. Campinas-SP: Editora da UNICAMP, 2005.

Nonaka, Ikujiro; Takeuchi, Hirotaka. Criação de conhecimento na empresa: como as empresas japonesas geram a dinâmica da inovação. 14 ed. Rio de Janeiro: Campus, 1997. 
Nooteboom, Bart. Learning and innovation in organizations and economies. Oxford: Oxford University Press, 2001.

Organização para cooperação econômica e desenvolvimento. Manual Oslo de Inovação. Oslo, Noruega, 1997.

Pavitt, Keith. Innovation process. In: Jan Fagerberg, David C. Mowery e Richard R. Nelson (Eds.). The Oxford Handbook of Innovation. Oxford: Oxford University Press, p. 86-114, 2005.

Pawlowsky, Peter. The treatment of organizational learning in management science. In: Meinolf Dierkes, Ariane Bertoihn Antal, John Child e Ikujiro Nonaka (Eds.). Handbook of organizational learning and knowledge. Oxford: Oxford University Press, p. 61-88, 2003.

Pellegrin, Ivan; Balestro, Moisés V.; Antunes Júnior, José Antonio Valle; Caullirax, Heitor Mansur. Redes de inovação: construção e gestão da cooperação pró-inovação. Revista de Administração, São Paulo, vol. 42, n. 3, p. 313-325, 2007.

Pérez, Manuela Pérez; Sánchez, Angel Martínez; Carnicer, Pilar De Luis; Jiménez, María José Vela. A technology acceptance model of innovation adoption: the case of teleworking. European Journal of Innovation Management, vol. 7, n. 4, p. 280-291, 2004.

Pisano, Gary P. Knowledge, integration, and the locus of learning: an empirical analysis of process development. Strategic Management Journal, vol. 15, p. 85-100, 1994.

Polanyi, Michael. Personal knowledge: towards a post-critical philosophy. London: Routledge, 1958.

PowelL, Walter W. Hybrid organizational arrangements: new form or transitional development? California Management Review, vol. 30, n. 1, p. 67-87, 1987.

Powell, Walter W.; Koput, Kenneth W.; Smith-Doerr, Laurel. Interorganizational collaroation and the locus of innovation: networks of learning in biotechnology. Administrative Science Quarterly, vol. 41, p. 116-145, 1996.

Prochno, Paulo. Transferindo práticas: construindo conhecimento arquitetural localmente. Revista de Administração de Empresas, vol. 44, n. 1, p. 70-81, 2004.

Ring, Peter Smith; VAN DE VEN, Andrew H. Developmental process of cooperative interorganizational relationships. Academy of Management Review, vol. 19, n. 1, p. 90-118, 1994.

Schumpeter, Joseph A. A teoria do desenvolvimento econômico. São Paulo: Nova Cultural, 1985.

Shrivastava, Paul. A typology of organizational learning systems. Journal of Management Studies, vol. 20, n. 1, p. 7-28, 1983.

Simon, Herbert A. Comportamento administrativo: estudo dos processos decisórios nas organizações administrativas. Rio de Janeiro: Editora da Fundação Getúlio Vargas, 1979. 
Spender, J.-C.; Grant, Robert M. Knowledge and the firm: overview. Strategic Management Journal, vol. 17, winter special issue, p. 5-9, 1996.

Starbuck, William H.; Hedberg, Bo L. T. How organizations learn from success and failure. In: Bo L. T. Hedberg, Meinolf Dierkes, Ariane Berthoin-Antal, John Child e Ikujiro Nonaka (Eds.). Handbook of Organizational Learning and Knowledge. Oxford: Oxford University Press, p. 327-350, 2001.

Staropoli, Carine. Cooperation in R\&D in the pharmaceutical industry: the network as an organizational innovation governing technological innovation. Technovation, vol. 18, n. 1, p. 13-23, 1998.

Steiner, Carol J. A philosophy for innovation: the role of unconventional individuals in innovation success. Journal of Product Innovation Management, vol. 12, p. 431-440, 1995.

Teece, David J.; Pisano, Gary; Shuen, Amy. Dynamic capabilities and strategic management. Strategic Management Journal, vol. 18, n. 7, p. 509-533, 1997.

Tsai, Wenpin. Knowledge transfer in intraorganizational networks: Effects of network position and absorptive capacity on business unit innovation and performance. Academy of Management Journal, vol. 44, n. 5, p. 996-1004, 2001.

Tsang, Eric W. K. Transferring knowledge to acquisition joint ventures: an organizational unlearning perspective. Management Learning, vol. 39, n. 1, p. 5-20, 2008.

Tushman, Michael; Nadler, David. Organizing for innovation. California Management Review, vol. 28, n. 3, p. 74-92, 1986.

Van Wijk, Raymond, JANSEN, Justin J. P.; LYLES, Marjorie. Inter- and intra-organizational knowledge transfer: a meta-analytic review and assessment of its antecedents and consequences. Journal of Management Studies, vol. 45, n. 4, p. 830-853, 2008.

Vargas, Eduardo Raupp; Zawislak, Paulo Antônio. Inovação em serviços no paradigma da economia do aprendizado: a pertinência de uma dimensão espacial na abordagem dos sistemas de inovação. Revista de Administração Contemporânea, vol. 10, n. 1, p. 139-159, 2006.

Vera, Dusya; Crossan, Mary. Organizational learning and knowledge: toward an integrative framework. In: Mark Easterby-Smith e Marjorie A. Lyles (Eds.). Handbook of organizational learning and knowledge management. Oxford: Blackwell Publishing, p. 122-141, 2005.

Von Krogh, Georg. Care in knowledge creation. California Management Review, vol. 40, n. 3, p.133-153, 1998.

Weick, Karl E.; Westley, Frances. Organizational learning: affirming an oxymoron. In: Stewart R. Clegg, Cynthia Hardy e Walter R. Nord (ed.) Handbook of organization studies. London: Sage, p. 440-458, 1996. 
Wolfe, Richard A. Organizational innovation: review, critique and suggested research directions. Journal of Management Studies, vol. 31, n. 3, p. 405-431, 1994.

Zietsma, Charlene; Winn, Monika; Branzei, Oana; Vertinsky, Ilan. The war of the woods: facilitators and impediments of organizational learning process. British Journal of Management, vol. 13, special issue, p. S61-S74, 2002.

\title{
KNOWLEDGE, LEARNING AND INNOVATION IN ORGANIZATIONS: A PROPOSAL FOR CONCEPTUAL INTERACTION
}

\begin{abstract}
Knowledge, learning and innovation in organizations are polysemic constructs and their literature is fragmented and dispersed. At the same time, service organizations have their own peculiarities that require a differentiated conceptual treatment of what is true for industrial organizations in general. The main purpose of this essay is to theoretically articulate the concepts of knowledge, learning and innovation in service organizations. To achieve this goal, each construct is at first defined, considering their specific singularities Then, the interfaces and limits between the constructs are discussed, highlighting their theoretical and practical similarities and differences. Using the economic learning approach, the Techno-Economic Paradigm and Innovation Services, the articulation among the three concepts is supported on the perspective of interaction among different stakeholders. Finally, it is suggested to consider knowledge, learning and innovation as interdependent concepts within the same thematic field for future research.
\end{abstract}

KEYWORDS: Knowledge in Organizations, Learning in Organizations, Innovation, Service Innovation, Organizational Studies.

Data do recebimento do artigo: 11/12/2009

Data do aceite de publicação: 27/05/2010

Revista de Administração e Inovação, São Paulo, v. 7, n. 2, p. 127-149, abr./jun. 2010 